\title{
Star-planet interactions
}

\section{Possibility of detecting the orbit-shrinking of a planet around a red giant}

\author{
Georges Meynet $^{1}$, Patrick Eggenberger ${ }^{1}$, Giovanni Privitera ${ }^{1}$, Cyril Georgy $^{1}$, Sylvia Ekström ${ }^{1}$, \\ Yann Alibert ${ }^{2}$, and Christophe Lovis ${ }^{1}$ \\ 1 Geneva Observatory, University of Geneva, Maillettes 51, 1290 Sauverny, Switzerland \\ e-mail: georges .meynet@unige.ch \\ 2 Physikalisches Institut \& Center for Space and Habitability, Universitaet Bern, 3012 Bern, Switzerland
}

Received 18 April 2017 / Accepted 26 May 2017

\begin{abstract}
The surface rotations of some red giants are so fast that they must have been spun up by tidal interaction with a close companion, either another star, a brown dwarf, or a planet. We focus here on the case of red giants that are spun up by tidal interaction with a planet. When the distance between the planet and the star decreases, the spin period of the star decreases, the orbital period of the planet decreases, and the reflex motion of the star increases. We study the change rate of these three quantities when the circular orbit of a planet of $15 M_{\mathrm{J}}$ that initially orbits a $2 M_{\odot}$ star at 1 au shrinks under the action of tidal forces during the red giant phase. We use stellar evolution models coupled with computations of the orbital evolution of the planet, which allows us to follow the exchanges of angular momentum between the star and the orbit in a consistent way. We obtain that the reflex motion of the red giant star increases by more than $1 \mathrm{~m} \mathrm{~s}^{-1}$ per year in the last $\sim 40 \mathrm{yr}$ before the planet engulfment. During this phase, the reflex motion of the star is between 660 and $710 \mathrm{~m} \mathrm{~s}^{-1}$. The spin period of the star increases by more than about 10 min per year in the last $3000 \mathrm{yr}$ before engulfment. During this period, the spin period of the star is shorter than $0.7 \mathrm{yr}$. During this same period, the variation in orbital period, which is shorter than $0.18 \mathrm{yr}$, is on the same order of magnitude. Changes in reflex-motion and spin velocities are very small and thus most likely out of reach of being observed. The most promising way of detecting this effect is through observations of transiting planets, that is, through changes of the beginning or end of the transit. For the relatively long orbital periods expected around red giants, long observing runs of typically a few years are needed. Interesting star-planet systems that currently are in this stage of orbit-shrinking would be red giants with fast rotation (above typically $4-5 \mathrm{~km} \mathrm{~s}^{-1}$ ), a low surface gravity $(\log g$ lower than 2), and having a planet at a distance typically smaller than about $0.4-1$ au, depending on $\log g$. A space mission like PLATO might be of great interest for detecting planets that are on the verge of being engulfed by red giants. The discovery of a few systems, even only one, would provide very interesting clues about the physics of tidal interaction between a red giant and a planet.
\end{abstract}

Key words. planet-star interactions - stars: rotation - planets and satellites: detection

\section{Introduction}

Red giant stars are a stellar evolutionary phase that is favorable for studying the exchange of angular momentum between a planetary orbit and its host star. The reason is that red giants are very slow rotators (most of them rotate more slowly than $3 \mathrm{~km} \mathrm{~s}^{-1}$ for surface gravities below about $\log g \sim 2$ ), and when they evolve along the red giant branch, they slow down. The observations of fast-rotating red giants (typically with a rotation faster than $8 \mathrm{~km} \mathrm{~s}^{-1}$, only a few percent are found) triggered the idea that these stars may have been spun up by tidal interaction with a companion, possibly followed by the engulfment of that companion (Livio \& Soker 1984a; Soker et al. 1984; Sackmann et al. 1993; Rasio et al. 1996; Siess \& Livio 1999a,b; Villaver \& Livio 2007; Sato et al. 2008; Villaver \& Livio 2009; Carlberg et al. 2009; Nordhaus et al. 2010; Kunitomo et al. 2011; Bear \& Soker 2011; Mustill \& Villaver 2012; Nordhaus \& Spiegel 2013; Villaver et al. 2014; Privitera et al. 2016b,c).

In previous works, we studied the evolution of orbits of planets of different masses around stars of various initial masses and rotations with different orbital periods (Privitera et al. 2016b). We determined the initial conditions required for an engulfment to occur during the red giant phase. We also computed the evolution of the star after the engulfment (Privitera et al. 2016c) and explored the possibility that the fast rotation of the red giant after an engulfment might produce an observable surface magnetic field (Privitera et al. 2016a). In the present work, we focus on the short phase during which the orbit is shrinking before the planet engulfment. During that phase, the red giant rotates ever faster, the orbital period decreases, and the reflex motion of the star accelerates. We wish to obtain some orders of magnitudes for these changes and see whether they are of sufficiently high amplitude during reasonable periods of times to be observed, eventually. If this were the case, this would offer extremely interesting clues about the physics of tides in such systems.

In Sect. 2 we briefly recall the main ingredients of our computations. The orbital evolution of a planet of 15 Jupiter mass $\left(M_{\mathrm{J}}\right)$ that orbits a $2 M_{\odot}$ star is presented in Sect. 3, together with the variations as a function of time of the reflex motion $\left(v_{\text {reflex,* }}\right)$, of the stellar spin period $\left(P_{\mathrm{rot}, *}\right)$, and of the planetary orbital period $\left(P_{\text {orb }}\right)$. In Sect. 4 we place these results into a broader perspective and give our conclusions.

\section{Stellar and orbital evolution models}

We used computations performed by Privitera et al. (2016b) that follow the evolution of the star and the evolution of the planetary 

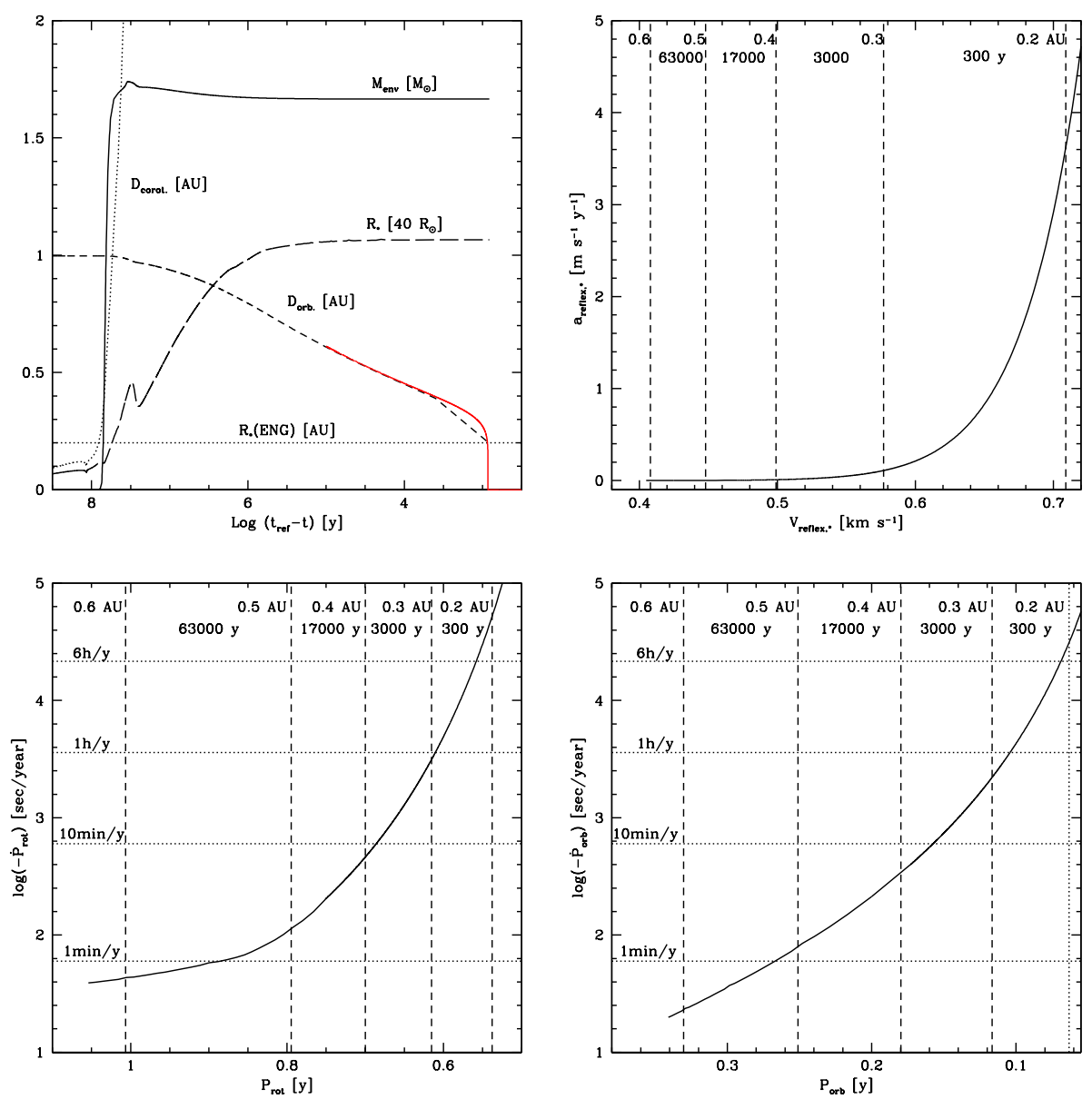

Fig. 1. Upper left panel: evolution as a function of time of a few relevant quantities during the last $100 \mathrm{Myr}$ before engulfment for a $15 M_{\mathrm{J}}$ planet orbiting a $2 M_{\odot}$ star $\left(\Omega_{\text {ini }} / \Omega_{\text {crit }}=0.1\right)$ at an initial distance equal to $1 \mathrm{au}$. The horizontal axis is the logarithm of the difference between a reference time $\left(t_{\text {ref }}\right)$ taken here equal to $1.29018 \mathrm{e}+09$ years and the age of the star. The short-dashed line labeled $D_{\text {orb }}$ shows the evolution of the semi-major axis of the orbit of the planet in units of au. Superimposed to this short-dashed line, the analytical solutions to the orbit given by Eq. (3) is plotted as a red continuous line. The dotted line labeled $D_{\text {corot }}$ is the corotation radius. The continuous lines $\left(M_{\text {env }}\right)$ and long-dashed line $\left(R_{\star}\right)$ show the evolution of the mass (in $M_{\odot}$ ) of the external convective envelope and of the radius of the star (in units of $40 R_{\odot}$ ). The horizontal dotted lines $\left(R_{\star}(\mathrm{ENG})\right)$ is the radius of the star at engulfment in units of au. Upper right panel: acceleration of the reflex motion of the star as a function of the reflex motion of the star during the last $83300 \mathrm{yr}$ before planet engulfment. The vertical lines show the periods corresponding to different radii of the orbit. The dotted line on the right indicates the period just before engulfment (engulfment occurs when the radius of the orbit is equal to the stellar radius, which is $0.2 \mathrm{au}$ ). The times in years correspond to the durations for passing from the orbit radius indicated to the left (see the labels of the vertical lines) to the radius indicated to the right of the times. Lower left panel: decrease of the stellar spin period as a function of the steallar spin period. Lower right panel: period variations during the planetary orbit-shrinking as a function of the orbital period.

orbit in a consistent way. By consistent, we mean here that the changes in orbit influence the stellar rotation through a boundary condition that allows angular momentum to be removed from or added to the star (depending on the sign of the tidal forces), and the change in planetary orbit accounts for the changes in the properties of the star (the tidal forces typically depend on the radius of the star and the size of the convective envelope, among other factors; see Eq. (1) below). Privitera et al. (2016b) provide all the details about the physical ingredients used to compute these models.

The evolution of the semi-major axis $a$ of the planetary orbit, which we assume to be circular $(e=0)$ and aligned with the equator of the star, accounts for the changes in the mass of the planet (by accretion of wind material or through evaporation), of the star (through stellar winds), for the effects of the circumplanetary material (drag forces), and for the tides (see Zahn 1966, 1977, 1989; Alexander et al. 1976; Livio \& Soker 1984b; Villaver \& Livio 2009; Mustill \& Villaver 2012; Villaver et al. 2014). When the tidal term dominates, other forces such as the drag forces play a negligible role, and we concentrate here on the tidal force. The evolution of the semi-major axis $a$ that is due to the tides is given by Zahn $(1966,1977,1989)$,

$$
(\dot{a} / a)_{\mathrm{t}}=\frac{f}{\tau} \frac{M_{\mathrm{env}}}{M_{\star}} q(1+q)\left(\frac{R_{\star}}{a}\right)^{8}\left(\frac{\Omega_{\star}}{\omega_{\mathrm{pl}}}-1\right),
$$

with $f=1$, when $\tau<P / 2$, and equal to $(P / 2 \tau)^{2}$ otherwise, where $P$ is the orbital period, $\tau$ is the eddy turnover timescale, which is taken as in Rasio et al. (1996). This factor allows us to consider only the convective cells that contribute to the viscosity (Villaver \& Livio 2009). $M_{\text {env }}$ is the mass of the stellar convective envelope, $M_{\star}$ is the stellar mass, $q=M_{\mathrm{pl}} / M_{\star}$ with $M_{\mathrm{pl}}$ is the mass of the planet, $\Omega_{\star}$ is the angular velocity at the surface of the star, and $\omega_{\mathrm{pl}}$ is the orbital angular velocity of the planet.

The inverse of the above equation gives a timescale that corresponds to the time for $a$ to change by a factor $e$. 


\section{Changes in reflex movement, spin, and orbital periods before planet engulfment}

We focus on a $15 M_{\mathrm{J}}$ mass planet orbiting a $2 M_{\odot}$ star at an initial distance of $1 \mathrm{au}^{1}$. We consider the case where the initial rotation of the star is equal to $\Omega / \Omega_{\text {crit }}=0.1$ (i.e., an initial velocity on the ZAMS equal to $32.2 \mathrm{~km} \mathrm{~s}^{-1}$ ). Privitera et al. (2016b) showed that for such a system planet engulfment occurs during the red giant phase.

We show in Fig. 1 (upper left panel) the temporal evolution of a few quantities during the last 100 My before planet engulfment. In particular, the dashed line shows the evolution of the semi-major axis of the orbit of the planet $\left(D_{\text {orb }}=a\right)$. To better understand what occurs, we adopt a simplified equation for the tidal torque given in Eq. (1):

$$
a^{7}(\dot{a})_{t}=-\underbrace{\frac{f}{\tau} \frac{M_{\mathrm{env}}}{M_{\star}} q(1+q) R_{\star}^{8}}_{C} .
$$

To obtain this equation, we assume that $\left(\frac{\Omega_{\star}}{\omega_{\mathrm{pl}}}-1\right) \sim-1$, that is, $\Omega_{\star}$ is significantly smaller than $\omega_{\mathrm{pl}}$, or $P_{\text {rot }}$ is longer than $P_{\text {orb }}$. This is indeed the case. Compare the abscissas of the two lower panels in Fig. 1. $P_{\text {rot }}$ is a factor 3-5 longer than $P_{\text {orb }}$ during the phase shown. During the last 100000 yr before engulfment, the right-hand term can be considered constant. In that case, the equation has an analytical solution given by

$$
a(t)=\left(a_{0}^{8}-8 C\left(t-t_{0}\right)\right)^{1 / 8},
$$

where $a_{0}$ is the radius of the orbit at the time $t_{0}$. By adopting values obtained at the time of engulfment as representative values for this whole period and plugging them into the analytic solution, we obtain the (red) continuous line in Fig. 1. There is a slight mismatch with the solution obtained in the complete numerical model following the evolution of the star and of the orbit (see the black dashed line) that is due mainly to the fact that stellar evolution computations prevent us from reducing the time steps as much as we would like. The analytic solution, on the other hand, while providing an excellent fit to the more complete numerical solution, allows us to describe the very last phases before the engulfment in much more detail. The shrinking of the planetary orbit strongly accelerates due to the ever increasing tidal forces when the distance to the star decreases.

In the upper right panel of Fig. 1, the change in the reflex motion of the star is plotted as a function of the reflex velocity. The reflex velocity is given by $v_{\text {reflex,* }}=\sqrt{G M_{*} / a} m_{\mathrm{pl}} / M_{*}$, with $G$ the gravitational constant. It can be written $v_{\text {reflex,* }} \approx$ $30 \sqrt{M_{*} / a} m_{\mathrm{pl}} / M_{*} \mathrm{~km} \mathrm{~s}^{-1}$, where the masses are in solar units and $a$ in au. For our case, this formula typically gives a velocity of around $0.580 \mathrm{~km} \mathrm{~s}^{-1}$ when $a$ is equal to $0.3 \mathrm{au}$. The acceleration of this reflex motion is given by $\mathrm{d} v_{\text {reflex,* }} / \mathrm{d} t=$ $-0.5 v_{\text {reflex,* }} \dot{a} / a$. The inverse of $\dot{a} / a$ is a time. An approximate value of it can be read in the right upper panel of Fig. 1, for instance, from the durations that are indicated at the top of the panel. The timescale is typically around $1500 \mathrm{yr}$ for $a$ equal to $0.3 \mathrm{au}$, which translates into an acceleration of the order of $20 \mathrm{~cm} \mathrm{~s}^{-1}$ per year. During the four decades before the engulfment, this acceleration reaches values higher than one meter per second per year. However, as discussed in Sect. 4, such low values are beyond reach because they are blurred by the jitter that is caused by convection in red giants.

\footnotetext{
By initial distance, we mean at a distance of 1 au on the ZAMS.
}

The variation in stellar spin is shown in the lower left panel of Fig. 1. The rotation period varies by more than about 10 minutes per year in the $3000 \mathrm{yr}$ preceding the engulfment. This corresponds to a relative change in the rotation period (around $0.65 \mathrm{yr}$ during that period) of more than $3 \times 10^{-5}$. This low value is beyond reach of current observations (see Sect. 4).

The most promising way of detecting the effects of the tides would probably be through the change in orbital period. The variation in orbital period is shown in the lower right panel of Fig. 1 for the last part of the evolution of the orbit. We see, for instance, that when the orbital period is around $0.18 \mathrm{yr}$, about $3000 \mathrm{yr}$ before the engulfment, the period change is about 5 min per year. Observing such a star for five years would accumulate data for nearly 28 revolutions, and the change between the first and the last orbital period would be of about $25 \mathrm{~min}$. Such a period difference is detectable for transiting planets for which accurate measurements of the variation of the beginning or end of the eclipse can be obtained.

How do these estimates change when the mass of the star, its rotation rate, the mass of the planet, and its initial distance to the star vary? It is beyond the scope of this letter to discuss these dependencies in details. This will be the topic of a more extended work. We content ourselves here with a few general remarks. It can be shown that the derivatives of $v_{\text {reflex,* }}, P_{\text {spin }}$ and $P_{\text {orb }}$ are proportional to $\dot{a} / a$, which is the inverse for the timescale of the orbit shrinkage. Thus the higher $\dot{a} / a$, the larger the derivatives, but on a shorter period. This is independent of the system characteristics. The quantity $\dot{a} / a$ is dominated by the term in $R_{*}^{8}$, thus by the radius of the star at the time of the engulfment. The time of the engulfment and thus this radius depends on the initial conditions considered. For a given initial mass of the star, the engulfment occurs later and thus at larger radius along the red giant branch for less massive planets with larger initial orbits (see Table A.1 in Privitera et al. 2016b). Thus the largest variations are expected for these systems. We note that the time of the engulfment depends weakly on the initial rotation of the star. $\dot{a} / a$ varies with $q$, the ratio between the mass of the planet to that of the star (since during the red giant phase $M_{\text {env }}$ is about $M_{*}$, we consider here that the factor $M_{\text {env }} / M_{*}$ is about 1$)$. Thus everything being equal, the variations are largest when $q$ is large. However, as indicated above, when $q$ is large, the engulfment occurs at earlier time when the red giant radius is smaller, making the variations smaller. This last effect in general dominates, and we therefore expect to see the largest variations for lower $q$ values. This means that the largest variations are expected for systems whose orbit shrinkage occurs near the top of the red giant branch. This shrinkage occurs near the top for systems with low $q$ values and for which the initial distance of the planet to the star is large.

\section{Discussion and conclusion}

Two great challenges need to be met to have a chance to see the variations discussed in this paper. First, we need very precise measurements at different times of the spin or orbital periods or of the reflex motion. Second, we need a careful selection of the red giants that are worthwhile to be monitored.

We discuss the first point. We have seen above that during the four decades before the engulfment, the reflex motion accelerates at a rate of more than one meter per second per year. While these quantities are small, they are not beyond reach of present facilities that can reach radial velocity measurement with a precision of the order of one meter per second (Mayor et al. 2003) and of future facilities such as ESPRESSO on the VLT, which will 
reach a precision of the order of $10 \mathrm{~cm} \mathrm{~s}^{-1}$ (Pepe et al. 2013). We note, however, that red giants have a large external convective zone with velocities of the convective cells that can reach values of up to a few $\mathrm{km} \mathrm{s}^{-1}$, which means that the detection of

The change in spin period of the star is of the order of $10 \mathrm{~min}$ per year in the last $3000 \mathrm{yr}$ before engulfment. The rotation period is shorter than $0.65 \mathrm{yr}$ during this period, which means that observing the star for five years would collect data for about 10 otations, and a change of nearly one hour would be found between the first and last spin period. This corresponds to a relative change $\left(\Delta P_{\text {spin }} / P_{\text {spin }}=-\Delta v_{\text {eq }} / v_{\text {eq }}\right.$, where $v_{\text {eq }}$ is the equatorial rotation velocity) of $2.3 \times 10^{-5}$. This is again a very delicate observation to be performed. Note that the velocity of the convective cells is of the same order of magnitude as the equatorial velocity and contributes to a similar amount to the enlargement of the absorption lines.

As we indicated above, the change in orbital period of a transiting planet is the most promising way of detecting the effect discussed here. Using transiting planets means that very accurate determinations of the period changes can be obtained, observing shifts of the beginning and/or end of the transit. The typically expected time shifts are of the order of about one hour or more in a time span of about five years during the last $23000 \mathrm{yr}$ before the engulfment. Some first period variations have been observed for planet-orbiting subgiants (Maciejewski et al. 2016; Patra et al. $2017)^{3}$. The case of subgiants is more favorable for detecting such effects. Subgiants have smaller radii, thus the planets can orbit with shorter orbital periods than around red giants. This allows accumulating many more orbital revolutions in a given time frame. On the other hand, at the subgiant phase, the signs of the star being a fast rotator as a result of some tidal interactions are more difficult to detect since during this phase the surface rotation of the star still depends strongly on the initial rotation (see Fig. 1 in Privitera et al. 2016b). This complicates the identifications of the interesting candidates. More evolved red giants, regardless of the initial rotation of the star, rotate so slowly that any rotation above some level might be used to identify candidates that are interesting for follow-up observations.

We now consider the selection of candidates to be monitored. Candidates that would fall in the frame of the computations shown here would present the following features: the host star should be a red giant with a $\log g$ below about 2 and a surface rotational velocity higher than $3-4 \mathrm{~km} \mathrm{~s}^{-1}$. A planet with at least one Jupiter mass should have been detected to orbit the star at a distance smaller than 1 au. There is some chance that such a system would be in the orbit-shrinkage phase, unless the high observed velocity were due to a previous planet engulfment. When we discard this last possibility and assume a duration for the orbit shrinkage of about $100000 \mathrm{yr}$, the period during which the variation amplitudes are about one hour or more over an observing time of five years would be about $3000 \mathrm{yr}$, which means about $3 \%$. This means that of 1000 red giants with the properties above, very roughly 30 should be in the shrinking period. The transit probability can easily be derived and is equal to $\sim R_{\star} / a$. In our case it amounts to $40 / 214 \sim 18.7 \%$, which means that between five and six might show a transit. Of course, the larger the such an effect is probably beyond reach ${ }^{2}$.

star, the more luminous it is, and thus also the more difficult it is to detect a transit.

About 100 red giants are known (the precise number is 99) that show the presence of a planet ${ }^{4}$. Nine of these consists of a star with a mass between 1.8 and $2.2 M_{\odot}$, that is, in the range of the case studied here, and have a planet at a distance shorter than $1 \mathrm{au}$. There is no case at the moment that would show both features ( $\log g$ smaller to 2 with $v_{\text {eq }}$ higher than $3-4 \mathrm{~km} \mathrm{~s}^{-1}$ ), which would be indicative of planetary orbit shrinking. A very interesting case is TYC 3667-1289b Niedzielski et al. (2016; $1.9 M_{\odot}$ star with a planet of $5.4 M_{\mathrm{J}}$ at least orbiting at a distance of $0.21 \mathrm{au}$ ). Interestingly, it has a chance of about $14 \%$ to present a transit. On the other hand, the rotation velocity of the star is not very high with a $v \sin i$ of $3.2 \mathrm{~km} \mathrm{~s}^{-15}$, therefore there is little chance that the planet is in a phase of fast orbital shrinking. However, it might be an interesting object to continue to observe, especially if it shows a transit. As we mentioned, the study of such systems would bring very interesting and probably unique constraints on the physics of tides. Measuring the timescale of the orbit shrinking for a few systems would allow us in particular to check the dependance of this timescale on the radius of the star, an important first step for assessing the physics of tides. This might be an interesting science case for a space mission like PLATO, which will be dedicated to transiting planets.

Acknowledgements. We thank the referee Eva Villaver for very valuable suggestions. The project has been supported by Swiss National Science Foundation grant 200020-172505. This work has been carried out in part within the frame of the National Centre for Competence in Research PlanetS supported by the Swiss National Science Foundation.

\section{References}

Alexander, M. E., Chau, W. Y., \& Henriksen, R. N. 1976, ApJ, 204, 879 Bear, E., \& Soker, N. 2011, MNRAS, 414, 1788

Carlberg, J. K., Majewski, S. R., \& Arras, P. 2009, ApJ, 700, 832

Kunitomo, M., Ikoma, M., Sato, B., Katsuta, Y., \& Ida, S. 2011, ApJ, 737, 66

Livio, M., \& Soker, N. 1984a, MNRAS, 208, 783

Livio, M., \& Soker, N. 1984b, MNRAS, 208, 763

Maciejewski, G., Dimitrov, D., Fernández, M., et al. 2016, A\&A, 588, L6

Mayor, M., Pepe, F., Queloz, D., et al. 2003, The Messenger, 114, 20

Mustill, A. J., \& Villaver, E. 2012, ApJ, 761, 121

Niedzielski, A., Villaver, E., Nowak, G., et al. 2016, A\&A, 589, L1

Nordhaus, J., \& Spiegel, D. S. 2013, MNRAS, 432, 500

Nordhaus, J., Spiegel, D. S., Ibgui, L., Goodman, J., \& Burrows, A. 2010, MNRAS, 408, 631

Patra, K. C., Winn, J. N., Holman, M. J., et al. 2017, ArXiv e-prints [arXiv: 1703.06582]

Pepe, F., Cristiani, S., Rebolo, R., et al. 2013, The Messenger, 153, 6 Privitera, G., Meynet, G., Eggenberger, P., et al. 2016a, A\&A, 593, L15 Privitera, G., Meynet, G., Eggenberger, P., et al. 2016b, A\&A, 591, A45 Privitera, G., Meynet, G., Eggenberger, P., et al. 2016c, A\&A, 593, A128 Rasio, F. A., Tout, C. A., Lubow, S. H., \& Livio, M. 1996, ApJ, 470, 1187 Sackmann, I.-J., Boothroyd, A. I., \& Kraemer, K. E. 1993, ApJ, 418, 457 Sato, B., Toyota, E., Omiya, M., et al. 2008, PASJ, 60, 1317

Siess, L., \& Livio, M. 1999a, MNRAS, 304, 925

Siess, L., \& Livio, M. 1999b, MNRAS, 308, 1133

Soker, N., Livio, M., \& Harpaz, A. 1984, MNRAS, 210, 189

Villaver, E., \& Livio, M. 2007, ApJ, 661, 1192

Villaver, E., \& Livio, M. 2009, ApJ, 705, L81

Villaver, E., Livio, M., Mustill, A. J., \& Siess, L. 2014, ApJ, 794, 3

Zahn, J. P. 1966, Ann. Astrophys., 29, 489

Zahn, J.-P. 1977, A\&A, 57, 383

Zahn, J.-P. 1989, A\&A, 220, 112

\footnotetext{
2 It should be noted, however, that convection produces a constant random noise, while the effect studied here consists of a shift.

3 We note that for these two cases, the authors tend to attribute the observed period changes to the tidal effects, although they do not exclude other causes.
}

4 See the catalog on the web https://www. lsw. uni-heidelberg. $\mathrm{de} /$ users/sreffert/giantplanets/giantplanets.php

5 If transit is detected and we have a coplanar planet, then this mean that this value is near the true equatorial velocity. 